Laboratory techniques

\title{
Plastic embedded core biopsy: a complementary approach to bone marrow aspiration for diagnosing acute myeloid leukaemia
}

\author{
A ISLAM,* BERTHA FRISCH, $\dagger$ E S HENDERSON* *Department of Medical Oncology, Roswell \\ Park Memorial Institute, Buffalo, New York, USA, and the †Institute of Haematology, Ichilov Hospital, Tel \\ Aviv, Israel
}

SUMMARY Bone marrow aspirates and biopsy specimens were taken at diagnosis from 51 patients with acute myeloid leukamia (AML). The diagnosis was based on morphological and cytochemical analyses, and the leukaemias were classified by FAB criteria. A considerable difference was observed between the results of bone marrow aspirates and the findings of plastic-embedded bone marrow biopsy specimens, particularly in marrow cellularity, extent of blast cell infiltration, and cell type involved in the leukaemic process. The myelomonocytic cell type seemed to predominate in the sections. In four cases there was considerable marrow infiltration with maturing, but dysplastic, granulocytic cells in the sections, but not in the aspirate smears. Features of potential prognostic importance, such as bone marrow infiltration with inflammatory cells, were easily recognised and quantified in the sections.

These results indicate that plastic embedded bone marrow biopsy sections complement the findings of bone marrow aspiration in the diagnosis of AML and may also provide information of independent prognostic importance that cannot be obtained by other means.

The diagnostic evaluation of bone marrow from patients with acute myeloid leukaemia (AML) is still done on a small marrow sample obtained by aspiration. These aspirates are diluted with sinusoidal blood, and may or may not accurately reflect the changes inherent in the leukaemic process or other structural changes of the marrow in these conditions. Cells anchored firmly within the marrow or situated focally may not be withdrawn on aspiration and thus can be excluded from examination. On the other hand, a long core of marrow provides a large volume of tissue, overcomes the problems associated with aspiration and, when processed into plastic, provides excellent cellular details and shows the structural organisation of the marrow. The haemopoietic cells can hence be evaluated in their own natural, undistorted environment, exhibiting the same cellular relation as in vivo. We report the findings of bone marrow aspirates and biopsy specimens, taken simultaneously from 51 patients with AML and classified according to the FAB criteria.

Accepted for publication 6 October 1988

\section{Patients and methods}

Fifty one patients with acute myeloid leukaemia (AML) were studied at the time of diagnosis. After informed consent had been obtained a marrow biopsy specimen, an aspirate, and a sample of peripheral blood were taken from each patient. AML was diagnosed from clinical, morphological, and cytochemical criteria according to the FAB classification. ${ }^{12}$

Air-dried smears of peripheral blood and bone marrow aspirates were fixed in methanol and stained with May-Grünwald and Giemsa's stain (MGG). The following cytochemical stains were also applied: periodic acid Schiff, Sudan black, myeloperoxidase, chloroacetate esterase, non-specific esterase, and acid phosphatase. Bone marrow cellularity was assessed by examining several individual marrow fragments in each case to establish the relative proportions of fat and marrow cells, and the overall marrow cellularity was expressed as a percentage.

Single bone marrow biopsy specimens were taken from the posterior iliac crest with a manual trephine $e^{34}$ and were processed in plastic (methyl-methacrylate) according to the methods previously described. ${ }^{56}$ 
Cytomorphologic classification based on plastic embedded bone marrow biopsy sections (Total no of cases $=51$ )

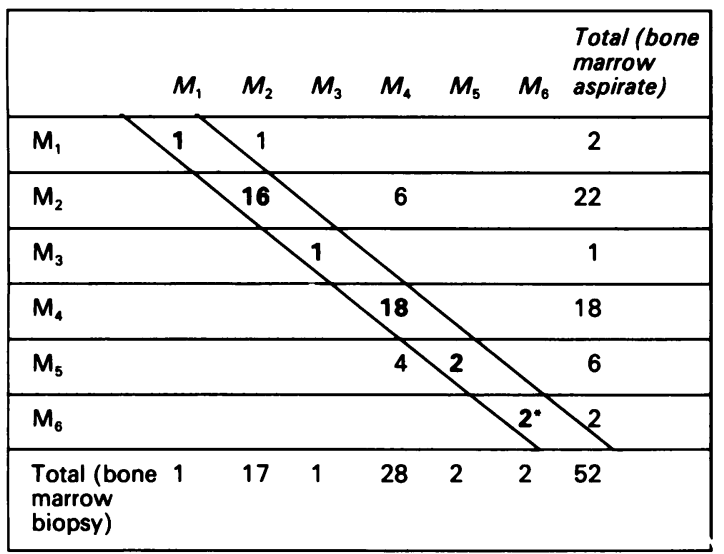

- One of these two cases showed involvement of megakaryocytes. The numbers in bold and within the tangential lines represent cases where there was concordance between bone marrow aspirate and biopsy specimen results.

Fig 1 Distribution of cases by fab classification.

Sections were cut at $2 \mu \mathrm{m}$ and stained with MGG stain $^{6}$ for cytological detail. Bone marrow cellularity in each section was assessed visually under low power and the overall marrow cellularity expressed as a percentage.

\section{Results}

Fig 1 shows the distribution of the 51 cases according to the FAB classification. The largest number of

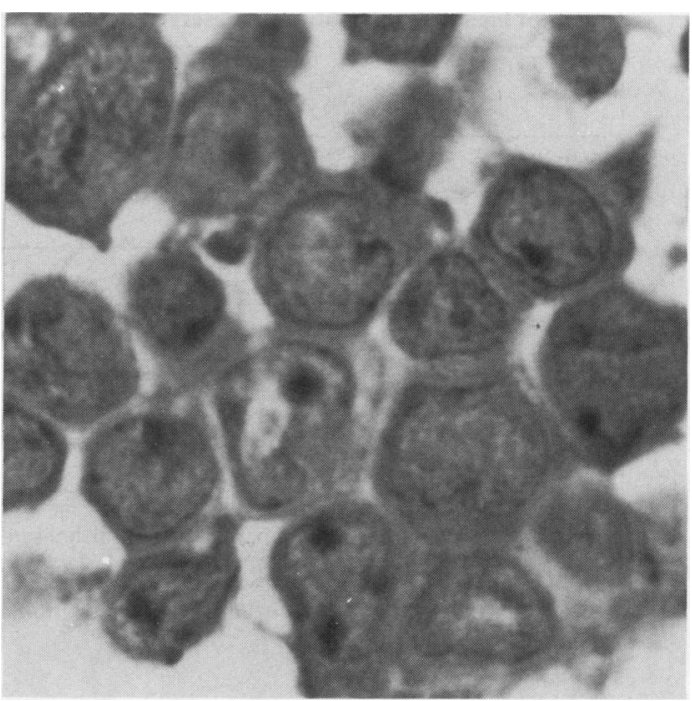

Fig 2 Bone marrow biopsy specimen ( $\left.F A B M_{2}\right)$ from a case of $A M L$ showing discrete cluster of monoblasts and promonocytes within marrow cavity. Note abundant cytoplasm, notched and indented nuclei, and prominent nucleoli in some cells. (Methacrylate, MGG stain.)

patients were in the M2 and M4 categories. When cytomorphological details were compared, differences in marrow morphology were observed between bone marrow aspirate and bone marrow biopsy specimen. One of the two cases classified as FAB M1 on the bone marrow aspirate showed small areas of mature granulopoietic cells in the section. Six of the 22 cases classified as FAB M2 and four of the six cases classified as FAB M5 showed some cells with morphological features of monoblasts and myelobolasts, respectively,

Bone marrow cellularity based on bone marrow biopsy section

\begin{tabular}{|l|c|cccc|}
\hline & $25<50 \%$ & $50<75 \%$ & $75<95 \%$ & $95-100 \%$ & $\begin{array}{c}\text { Total (bone } \\
\text { marrow aspirates) }\end{array}$ \\
\hline $10<25 \%$ & $10<25 \%$ & 0 & & 4 & 0 \\
\hline $25<50 \%$
\end{tabular}

The numbers in bold and within the tangential lines represent cases where there was concordance between bone marrow aspirate and biopsy specimen results.

Cohen's kappa $=0.14 p<0.001$

Fig 3 Comparison of marrow cellularity based on bone marrow aspirate and bone marrow biopsy specimen. 
Extent of blast cell infiltration as observed in bone marrow biopsy section.

\begin{tabular}{|c|cccccc|}
\hline & $20<40 \%$ & $40<60 \%$ & $60<80 \%$ & $>80 \%$ & $\begin{array}{c}\text { Total (bone } \\
\text { marrow aspirates) }\end{array}$ \\
\hline$<20 \%$ & 5 & 3 & 4 & 2 & 15 \\
\hline $20<40 \%$ & 1 & 1 & 1 & 1 & 3 & 6 \\
\hline $40<60 \%$ & 1 & 1 & 5 & 5 & 4 & 16 \\
\hline $60<80 \%$ & & & & 1 & 4 & 4 \\
\hline$>80 \%$
\end{tabular}

The numbers in bold and within the tangential lines represent cases where there was concordance between bone marrow aspirate and biopsy specimen results.

Fig 4 Comparison of extent of blast cell infiltration between bone marrow aspirate and bone marrow biopsy specimen.

in the sections. The distribution of blast cells with monocytoid features was not uniform and they were often seen in discrete clusters (fig 2) or near the paratrabecular regions. One of the two cases of erythroleukaemia (FAB M6) showed large clusters of dysplastic megakaryocytes in the sections but not in the smears.

In most cases bone marrow cellularity in sections was higher than that estimated in the aspirate smears (fig 3). In nine cases aspirate cellularity was less than $50 \%$; while in the sections it was over $50 \%$ in eight cases and over $95 \%$ in four of these eight cases. Concordance between the bone marrow aspirate and biopsy specimen cellularity was observed in only one case in this group. In 26 cases bone marrow aspirate cellularity ranged between $50-75 \%$; in sections 16 of the 26 cases showed a cellularity of $95-100 \%$ and in another case it ranged between $75-90 \%$. Concordance between the aspirate and biopsy specimen cellularity was observed in only nine of these 26 cases. In 16 cases bone marrow aspirate cellularity ranged between 75$90 \% ; 12$ of the 16 cases showed a cellularity of over $95 \%$ in the section. The statistical analysis ${ }^{7}$ of these results showed that there was more agreement than expected from chance alone $(p<0.001)$, but the degree of agreement was poor $(p<0 \cdot 14)$.

A considerable difference in the extent of blast cell infiltration was also observed between the aspirate smears and biopsy sections (fig 4). In a high proportion of cases the extent of blast cell infiltration was higher in sections than in the aspirate smears.

In about $50 \%$ of the cases the bone marrow biopsy specimen showed a dense infiltration of the marrow with a homegeneous population of immature (blast) cells (fig 5a); while in about the same number of cases the marrow populations were less homogeneous and contained a mixture of immature and more mature haematopoietic elements and inflammatory cells (fig 5b). A small proportion (four cases) of these cases also showed diffuse or patchy, but clinically important, marrow infiltration with maturing, but dysplastic, granulocytic cells (fig 6), which was not reflected in smears of the aspirates. The distribution pattern of megakaryocytes was affecied and they were either widely scattered or localised in small isolated clusters (fig 7), while they were often not found in the aspirate smears. Residual erythropoiesis, when present, was seen in small islands but was noticeably absent in the smears.

In 34 cases the marrow was infiltrated either alone or in combination with a variable number of inflammatory cells (lymphocytes, plasma cells, tissue mast cells, eosinophilic granulocytes and macrophages) (fig 8 ). The presence and the number of such inflammatory infiltrates could not be accurately assessed in the smears because of their patchy, compartmentalised, and non-uniform distribution pattern, and differential aspiratibility of the bone marrow aspirate.

\section{Discussion}

In recent years major advances have been made in the diagnosis and classification of $\mathrm{AML}^{28-10}$ though both are based primarily on the morphological cytochemical, cytogenetic and immunological characteristics of populations of blast cells present in a sample of marrow obtained by aspiration. In general, these aspirate samples are small and not truly representative of the marrow, and sampling errors inherent in the bone marrow aspiration technique remain a major problem. On the other hand, a long core marrow biopsy specimen overcomes these problems and 


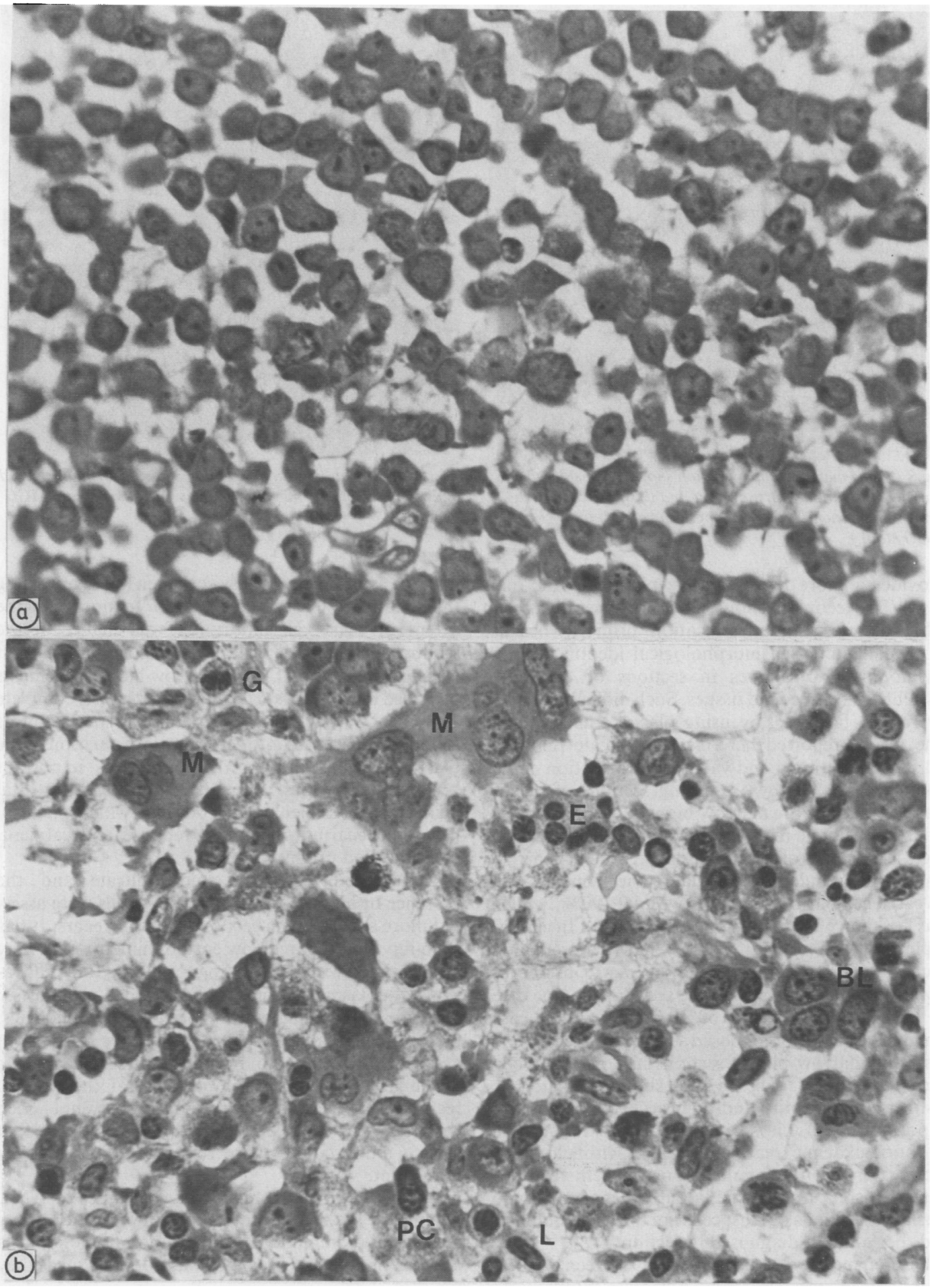

Fig 5 Bone marrow biopsy section from a case of AML showing (a) homogeneous infiltration of marrow with blast cells. Note presence of sheets of blasts and absence of mature haematopoietic cells; (b) section from another case of AML showing inhomogeneous marrow infiltration with blast cells. Note presence of leukaemic blasts intermixed with mature haematopoietic elements and inflammatory cells. $B L=$ blast cells, $M=$ megakaryocytes, $E=$ erythroid precursors, $G=$ granuloid precursors, $L=$ lymphocytes, $P C=$ plasma cells. (Methacrylate, MGG stain.) 


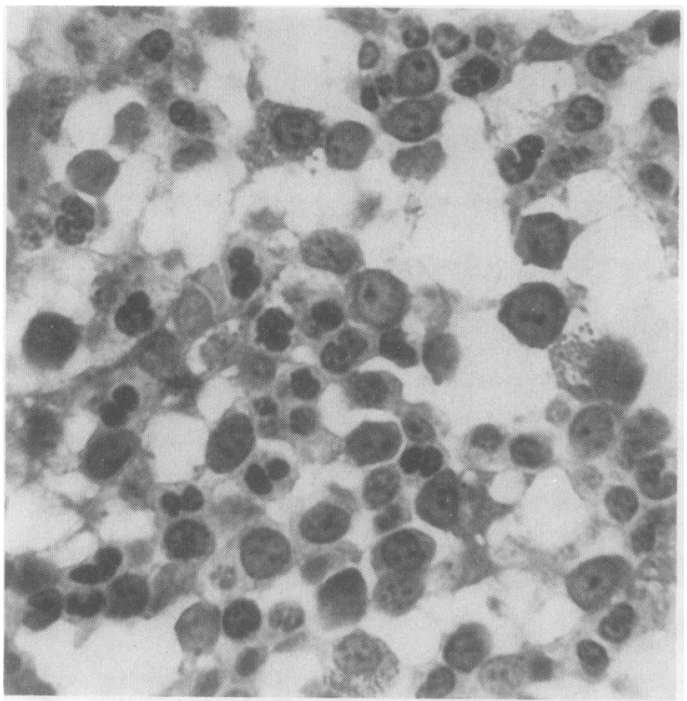

Fig 6 Bone marrow biopsy section showing considerable marrow infiltration with maturing, but dysplastic (pseudo Pelger-Huet anomaly) granulocytic cells. (Methacrylate, MGG stain.)

provides a more representative picture of the marrow. " This technique, however, is not generally used in AML diagnosis and classification, primarily because of the difficulties in morphological identification of haemopoietic cell types in sections of decalcified paraffin wax embedded tissues. Such difficulties can be overcome, however, by using plastic embedding. ${ }^{1213}$ The results reported here clearly indicate that considerable additional and valuable information can be obtained in AML by examining sections of plasticembedded long core marrow biopsy specimens.

Using the bone marrow biopsy specimen technique (fig 3), most (32 of 51 cases) pre-treatment marrows were highly cellular with virtual replacement of fat. In 18 cases cellularity ranged between $50-90 \%$, while it was less than $50 \%$ in only one case $(<2 \%)$. In general, aspirates did not reflect the marrow cellularity accurately and, in most cases, it was considerably lower than that observed in the sections. In nine cases $(>17 \%)$ it was less than $50 \%$. Some of these cases could have been misdiagnosed as hypoplastic $A M L^{14}$ if the bone marrow biopsy sections had not been concurrently studied. The hypoplastic AML's form a distinct clinical entity and their accurate diagnosis is important because it is generally accepted that intensive antileukaemic treatment shold be withheld for the group,${ }^{14}$ or that these patients may benefit from low dose cytosine arabinoside. ${ }^{15}$ Patchy marrow hypoplasia with islands of blast cells are the usual findings ${ }^{16}$ and they are best diagnosed in sections. ${ }^{17}$

Discrepancies were also observed between the bone

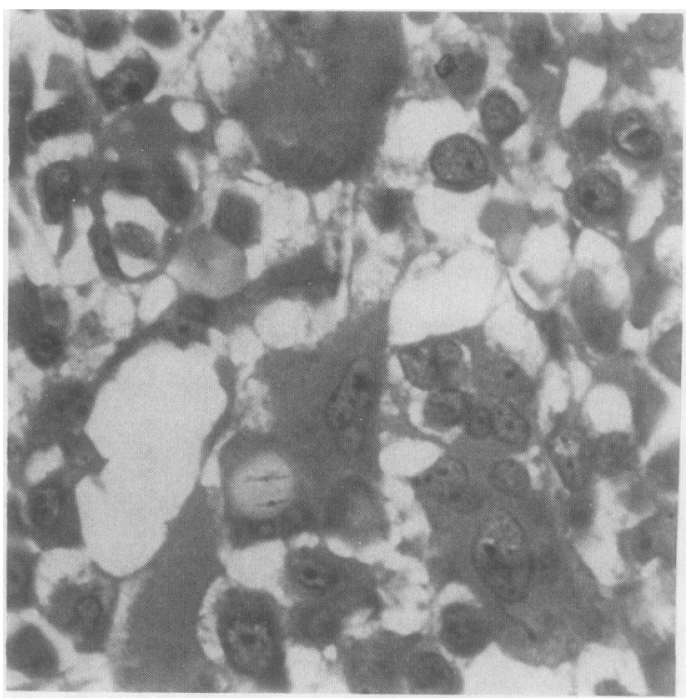

Fig 7 Bone marrow biopsy section showing a small isolated cluster of dysplastic megakaryocytes. (Methacrylate, MGG stain.)

marrow aspirate and biopsy specimen (fig 4) regarding the quantity of blast cell infiltration. In most cases the extent of blast cell infiltration was higher in the sections than in the aspirates, although in three cases it was lower. In general, bone marrow biopsy specimens provided more accurate results; nevertheless, complete agreement between aspirate and biopsy specimen was found in 12 cases $(25 \%)$. An accurate evaluation of the blast cell infiltration in the marrow is important because it provides a rough estimate of the total leukaemic mass (leukaemia burden) which, in turn, has some bearing on patient reponse to treatment, outcome, and prognosis.

Homogeneity of leukaemic infiltrate and the presence or absence of inflammatory cells were assessed more accurately in sections than in smears. It has been reported that marrow eosinophilia in AML may be associated with a good prognosis ${ }^{18}$ and that infiltration with plasma cells may indicate a bad prognosis. ${ }^{17}$ Increases in the inflammatory infiltrates (lymphocytes, plasma cells, and macrophages) have also been associated with a poor prognosis in severe aplastic anaemia. ${ }^{19}$ To date, the importance of these AML features in relation to prognosis has not been thoroughly investigated, and our results indicate that such a study could prove rewarding and clinically important.

Among the various morphological types of AML recognised in the section the myelomonocytic variety as the most common. The distribution of monoblasts and promonocytes in sections was not uniform as they 


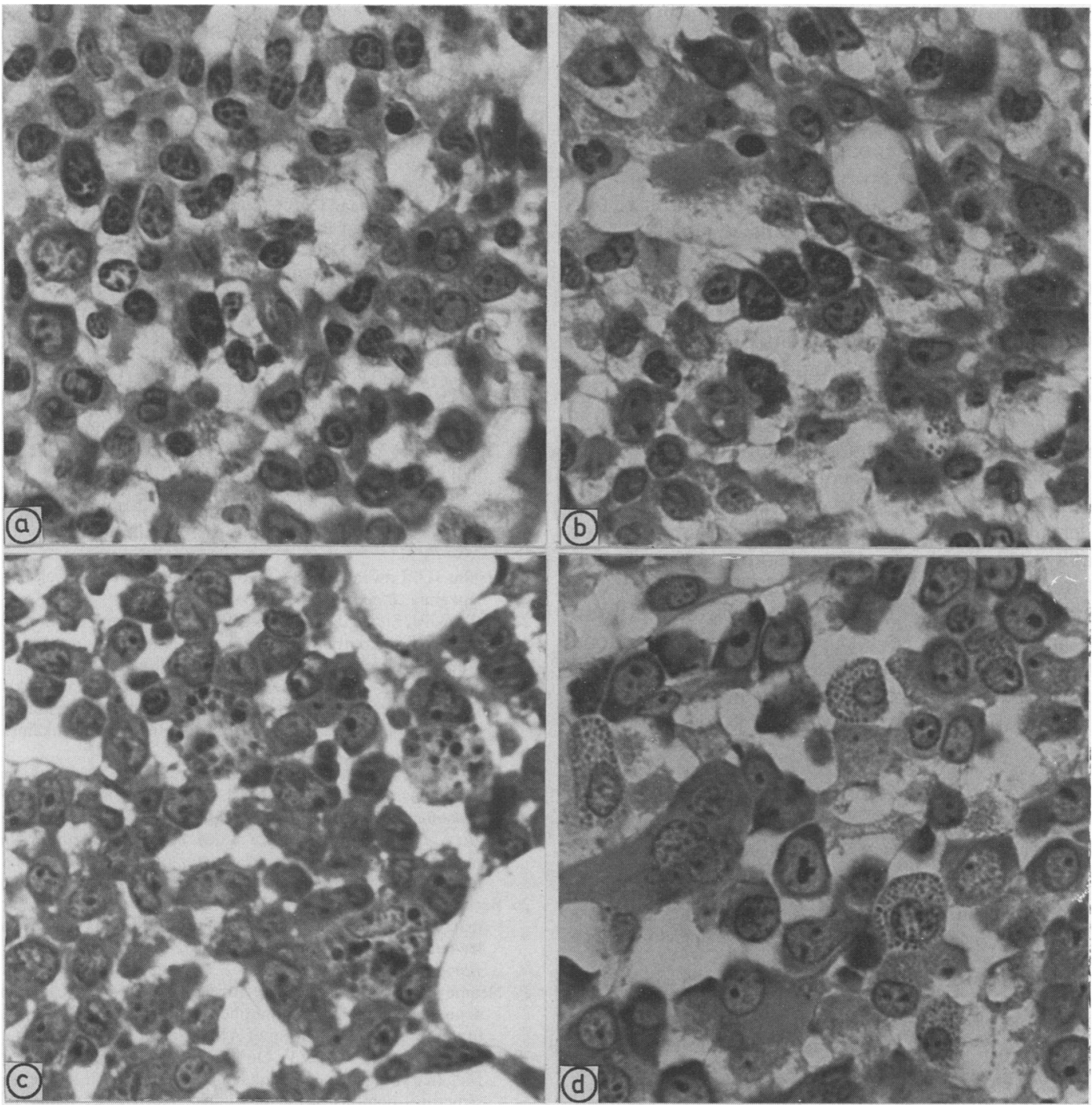

Fig 8 Details of bone marrow biopsy sections showing presence of inflammatory infiltrates; (a) lymphocytes, (b) plasma cells, (c) macrophages (d) eosinophilic granulocytes (Methacrylate, MGG stain.)

were often seen as small clusters or near the bony trabeculae. In one case of erythroleukaemia the presence of megakaryocytes seen in the sections was not apparent in the smears. The absence of megakaryocytes and monocytic elements in the smears may be an example of sampling error and selective resistance to marrow aspiration. The actual presence of these cells may also support the contention that AML is a proliferation of at least two and possibly all haemopoietic cell lines. ${ }^{20-23}$

An important finding of this study was the demonstration of a group of patients who had clinically important marrow infiltration with maturing,but dysplastic, granulocytic cells. This finding suggests that these patients may have undergone leukaemic transformation of a previously unrecognised myelodysplastic syndrome, or that they may represent a group of patients who present in blastic crisis of chronic myeloid leukaemia without a previously recognised chronic phase. Alternatively, they may also represent a group of patients in whom there is an abortive or residual attempt at differentiation in genuine AML. An accurate recognition of this group of patients is not only important from a therapeutic point of view, but 
also prognostically, as the presence of this group may affect the overall outcome of an AML protocol evaluation.

Our study shows that the guidelines proposed by the FAB cooperative group are useful in classifying most AML cases. It is also apparent from our study, as well as from others,${ }^{20}$ however, that maximal accuracy in categorisation of such conditions can be obtained only by analyses based on combinations of morphological and cytochemical, ${ }^{1224}$ histological and histochemical, ${ }^{17202526}$ immunological, ${ }^{927}$ karyotypical, ${ }^{1027}$ and ultrastructural ${ }^{2829}$ investigations. The recently developed technique ${ }^{23}$ of simultaneously identifying a cell as malignant (clonal chromosomal abnormalities) and determining its lineage by using lineage specific monoclonal antibodies could be very important in this regard. Immunohistological techniques now being developed for plastic embedded bone marrow biopsy specimens $\mathrm{s}^{26}$ may also add considerably to what can be learned from these techniques, most of which rely heavily on a sample of marrow obtained by aspiration. Through this immunohistological technique it would also be possible to characterise the leukaemic subpopulations that are localised or firmly anchored within special regions of the marrow and thus permit recognition of homogeneity or diversity in a leukaemic cell population.

\section{References}

1 Bennett JM, Catovsky D, Daniel MT, et al. Proposals for the classification of the acute leukemias. Br J Haematol 976;33: 451-8.

2 Bennett JM, Catovsky D, Daniel MT, et al. Proposed revised criteria for the classification of acute myeloid leukaemia: A report of the French-American-British Cooperative Group. Ann Intern Med 1985;103:620-9.

3 Islam A. A new bone marrow biopsy needle with core securing device. J Clin Pathol 1982;35:359-64.

4 Jamshidi K, Windschitl HE, Swaim ER. A new biopsy needle for bone marrow. Scand J Haematol 1971;8:69-71.

5 Burkhardt R. Bone marrow and bone tissue. In: Colour atlas of clinical histopathology. Berlin: Springer-Verlag 1971.

6 Islam MA, Frisch B. Plastic embedding in routine histology: I. Section preparation from undecalcified marrow core. Histopathol 1985;9:1263.

7 Fleiss JL. Statistical methods for rates and proportions. New York: John Wiley and Sons, 1981.

8 Bennett JM, Catovsky D, Daniel MT, et al. Characterisation of megakaryoblastic leukaemia (M7). Ann Intern Med 1985; 103:460-2.

9 Foon KA, Todd RF. Immunologic classification of leukaemia and lymphoma Blood 1986;68:1-31.

10 Second MIC Cooperative Study Group. Morphologic, immunologic, and cytogenetic (MIC) working classification of acute myeloid leukemias. Cancer Genet Cytogenet 1988;30:1-15.

11 Islam A, Henderson ES. Value of long-core biopsy in the detection of discrete bone-marrow lesions. Histopathol 1988;12:641-8.

12 Burkhardt R, Frisch B, Bartl R. Bone marrow biopsy in haematological disorders. J Clin Pathol 1982;35:257.

13 Frisch B, Lewis SM, Burkhardt R, Bartl R. Biopsy pathology of bone and marrow. London: Chapman \& Hall, 1985.

14 Needleman SW, Burns P, Dick FR, Armitage JO. Hypoplasic acute leukaemia. Cancer 1981;48:1410-14.

15 Manoharan A. Low-dose cytarabine therapy in hypoplasic acute leukemia. N Engl J Med 1983;309:1652-3.

16 Beard MEJ, Bateman CJT, Crowther DC, et al. Hypoplastic acute myelogenous leukaemia. Br J Haematol 1975;31:167-76.

17 Islam A, Catovsky D, Goldman JM, Galton DAG. Bone marrow biopsy changes in acute myeloid leukaemia I: observations before chemotherapy. Histopathol 1985;9:939-57.

18 Keating MJ. Factors influencing disease free interval after achieving complete remission in acute leukemia. In: Minimal residual disease in acute leukaemia. The Hague: Martinus Nijhoff, 1984:149-54.

19 Te Velde J, Haak HL. Aplastic anaemia: histological investigation of methacrylate embedded bone marrow biopsy specimens; correlation with survival after conventional treatment in 15 adult patients. Br J Haematol 1977;35:61-9.

20 Glick AD, Panker K, Flexner JM, Graber SE, Collins RD. Acute leukemia of adults. Ultrastructural, cytochemical and histological observations in 100 cases. Am J Pathol 1980;73: 459-70.

21 Griffin JD, Lowenberg B. Clonogenic cells in acute myeloblastic leukemia. Blood 1986;68:1185-95.

22 Fialkow PJ, Singer JW, Raskind WH, et al. Clonal development, stem-cell differentiation, and clinical remissions in acute nonlymphocytic leukemia. N Engl J Med 1987;317:468-73.

23 Keinanen M, Griffin JD, Bloomfield CD, Machnicki J, De La Chapelle A. Clonal chromosomal abnormalities showing multiple-cell-lineage involvement in acute myeloid leukemia. $N$ Engl J Med 1988;318:1153-8.

24 Flandrin G, Daniel MT. Cytochemistry in the classification of leukemias. In: Catovsky D, ed. Methods in hematology. The leukemic cell. Edinburgh: Churchill Livingstone, 1981:29-48.

25 Islam A, Henderson ES. Glycol methacrylate embedding for light microscopy I enzyme histochemistry on semithin sections of undecalcified marrow cores. J Clin Pathol 1987;40:1194-200.

26 Beckstead JH, Bainton D. Enzyme histochemistry on bone marrow biopsies: reaction useful in the differential diagnosis of leukemia and lymphoma applied to 2 -micron plastic sections. Blood 1980;55:386-93.

27 Neame PB, Soamboonsrup P, Browman GP, et al. Classifying acute leukemia by immunophenotyping: a combined FABimmunologic classification of AML. Blood 1986;68:1355-62.

28 Breton-Gorius J, Reytes F, Duhamel G, Najman A, Gorin NC Megakaryoblastic acute leukaemia: identification by the ultrastructural demonstration of platelet peroxidase. Blood 1978; 51:45-60.

29 Matutes E, Pombo de Oliveira M, Fornoni L, Morilla R, Catovsky $D$. The role of ultrastructural cytochemistry and monoclonal antibodies in clarifying the nature of undifferentiated cells in acute leukaemia. Br J Haematol 1988;69:205-11.

30 Islam A, Archimbaud E, Henderson ES, Han T. Glycol methacrylate (GMA) embedding for light microscopy. II. Immunohistochemstry on semithin sections of undecalcified marrow cores. J Clin Pathol 1988;41:892-6.

Requests for reprints to: Dr Anwarul Islam, Roswell Park Memorial Institute, 666 Elm Street, Buffalo, New York 14263, USA 